\title{
Highly Conducting Nanographite-Filled Paper Fabricated via Standard Papermaking Techniques
}

Patrik Isacsson, Xin Wang, Andreas Fall, Desalegn Mengistie, Emilie Calvie, Hjalmar Granberg, Göran Gustafsson, Magnus Berggren,* and Isak Engquist*

Cite This: ACS Appl. Mater. Interfaces 2020, 12, 48828-48835

Read Online

ABSTRACT: Eco-friendly and cost-effective materials and processes to manufacture functional substrates are crucial to further advance the area of printed electronics. One potential key component in the printed electronics platform is an electrically functionalized paper, produced by simply mixing common cellulosic pulp fibers with high-performance electroactive materials. Herein, an electronic paper including nanographite has been prepared using a standardized and scalable papermaking technique. No retention aid was needed to achieve a conducting nanographite loading as high as $50 \mathrm{wt} \%$. The spontaneous retention that provides the integrity and stability of the nanographite paper, likely originates partially from an observed water-stable adhesion of nanographite flakes onto the fiber surfaces. The resulting paper

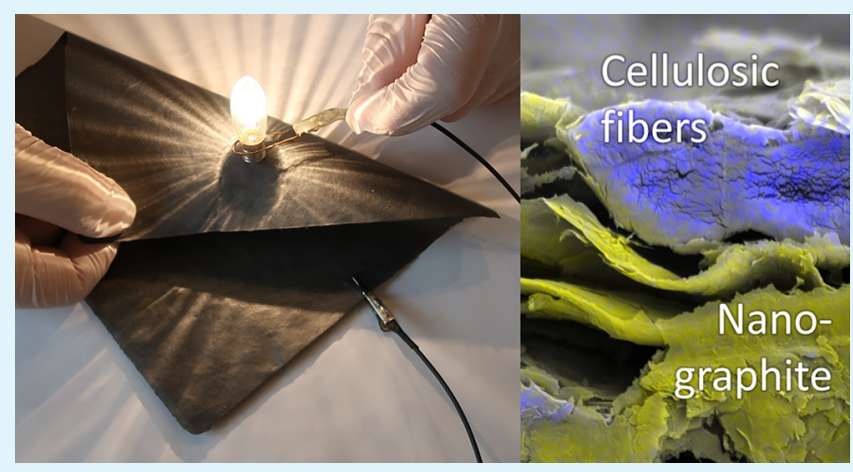
exhibits excellent electrical characteristics, such as an in-plane conductivity of $107 \mathrm{~S} / \mathrm{cm}$ and an areal capacitance of $9.2 \mathrm{mF} / \mathrm{cm}^{2}$, and was explored as the back-electrode in printed electrochromic displays.

KEYWORDS: electronic paper, printed electronics, electrochromic display, nanographite, graphene, cellulose, self-assembly

\section{INTRODUCTION}

The science and industry of printed electronics have made significant progress over the last decades, although reducing the environmental impact and the material costs are considered increasingly critical issues that must be dealt with. ${ }^{1}$ Therefore, biobased and inexpensive cellulosic paper as the substrate for printed electronics has attracted more and more interest from the sector, which is further motivated by the many production protocols that the industries of graphics and printed electronics have in common. ${ }^{2}$ Furthermore, it would be of interest to print on papers not only providing a printing substrate but also offering electronic functionality. For example, in electrochromic displays (ECDs), a conductive substrate could simultaneously serve as the flexible rear side substrate, the current collector, and the electrode (Figure 1A,B). ${ }^{3}$

Conductive cellulose-based composites have received much attention over the past decade, where cellulose nanofibrils (CNFs) have been successfully combined with nanocarbons. CNFs have an intrinsic attraction to nanocarbons, ${ }^{4,5}$ by which the excellent mechanical properties of CNFs and the high electrical conductivity of various nanocarbons can be exploited to prepare high-performing composites ${ }^{6,7}$ with conductivities approaching $10^{3} \mathrm{~S} / \mathrm{cm}^{8}{ }^{8}$ However, CNF-based composites are challenging to produce on a larger scale due to the inherent high dewatering resistance of CNFs. ${ }^{9}$ Ordinary papers based
A

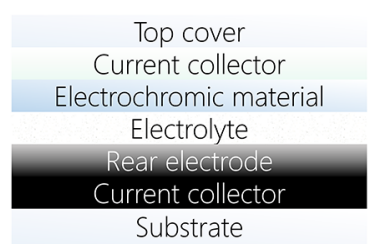

B

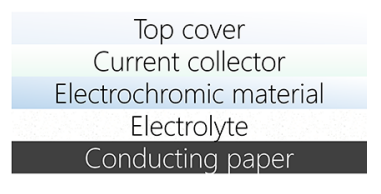

Figure 1. Scheme of the layered structure in a (A) standard and (B) simplified printed ECD, the latter using a multifunctional conducting paper like the NG paper reported in this work.

on macroscopic cellulosic pulp fibers are, on the contrary, produced in enormous quantities globally. Recently, it has been shown that bulk electronic functionality can be introduced in such papers. For instance, cellulosic pulp can be mixed with conducting graphite, which results in composites exhibiting both conductive and capacitive performance. ${ }^{10,11}$

Received: July 20, 2020

Accepted: October 2, 2020

Published: October 14, 2020 
However, it is not possible to translate the preparation of paper in vacuum funnels with fine filter media, as utilized in the previously mentioned works, into industrial papermaking processes. To realize the potential of electronic papers, we need a fabrication technique that allows for the large mesh size used in traditional paper machines and the demand for high rates of dewatering. ${ }^{12}$ Attempts to use standard papermaking techniques have, in fact, been explored to prepare conducting papers functionalized with conducting polymers; ${ }^{13,14}$ however, the attempts have only resulted in very low conductivities. By instead combining carbon fibers with cellulosic pulp fibers, a relatively high conductivity of $105 \mathrm{~S} / \mathrm{cm}$ has been achieved, ${ }^{15}$ although for this, a carbon fiber content of $80 \mathrm{wt} \%$ was needed. From a printed electronics perspective, the use of carbon fibers gives drawbacks related to poor mechanical performance as well as an open and porous structure not suitable for printing or other common converting steps. Thus there is still no example of a highly conducting paper that is producible in an up-scalable way with standard papermaking methods and exhibits good mechanical properties.

In this work, we have focused on exploring the combination of cellulosic pulp fibers and flaky, high-conducting carbon filler material in a standardized papermaking protocol. Instead of using bleached pulp, as was previously utilized in filled conducting papers, ${ }^{10,11}$ we use unbleached pulp of electrotechnical grade (treated to remove monovalent metal ions). This is motivated by the fact that the bleaching process significantly reduces the charge density of the fiber surface, ${ }^{16}$ which might lower any dispersing ability of the cellulosic fibers. ${ }^{17}$ In addition, for several reasons, we here select nanographite (NG) flakes in aqueous dispersions stabilized by poly(acrylic acid) (PAA) instead of utilizing carbon fibers or nanotubes as a filler material. First, NG flakes, derived from exfoliated graphite, are a promising low-cost carbon material that exhibits a high conductivity. ${ }^{18}$ Second, because their overall planar shape is expected to provide improved retention, ${ }^{19} \mathrm{NG}$ flakes should be preferable as compared with carbon nanotubes and fibers. Third, for the targeted application, it is desired to incorporate a conducting medium that provides enough active surface area for electrical charge storage.

The resulting NG paper exhibited good electrical conductivity and, at the same time, the desired mechanical characteristics of ordinary paper. We explored these properties by utilizing the NG paper as the combined counter electrode, rear current collector, and substrate for an all-printed ECD.

\section{EXPERIMENTAL SECTION}

Sample Preparation. NG from exfoliated graphite, produced by tube-shear exfoliation in water stabilized by PAA, as described by Blomquist et al., ${ }^{18}$ was kindly supplied by 2Dfab (Sundsvall, Sweden) with a solid content of $3.3 \%$. Never-dried unbleached kraft pulp of electrotechnical grade (UKP-E) was kindly supplied by AhlstromMunksjö Billingsfors mill (Billingsfors, Sweden). The wet aqueous mix solely contained UKP-E and NG with PAA.

The never-dried UKP-E pulp was diluted to $1.5 \%$ fiber content using local tap water and stirred for $20 \mathrm{~min}$ before measuring $1.0 \mathrm{~g}$ dry weight of fibers into a beaker. Separately, the NG dispersion was stirred for $20 \mathrm{~min}$ before pouring an equivalent of $2.0 \mathrm{~g}$ dry weight of NG into a beaker. The two dispersions were put into a blender and mixed for $2 \mathrm{~min}$. The UKP-E/NG ratio of 1:2 was chosen arbitrarily, although a ratio of $1: 3$ also was tested with no clear increase in conductivity.
The papers were made according to a standard protocol for laboratory handsheet preparation (ISO 5269-1) using a Lorentzen \& Wettre F1 101 sheet former, followed by wet pressing at $400 \mathrm{kPa}$ between blotters and spanned drying on a slightly convex metal surface. Using the same procedure, reference sample papers were prepared from $1.0 \mathrm{~g}$ of UKP-E.

The drainage resistance for the 1:2 UKP-E/NG mix as well as for pure UKP-E was assessed by the Schopper-Riegler method (ISO 5267-1).

Material Characterization. WAXS (wide-angle X-ray scattering) measurements were performed on an Anton Paar SAXSpoint 2.0 system equipped with a Microsource X-ray source and a Dectris $2 \mathrm{D}$ CMOS Eiger R $1 \mathrm{M}$ detector with a $75 \mu \mathrm{m} \times 75 \mu \mathrm{m}$ pixel size. All measurements were performed with a beam size of $\sim 500 \mu \mathrm{m}$ diameter and a pressure of about 1 to $2 \mathrm{mbar}$ at a temperature of $25^{\circ} \mathrm{C}$. The evaporated NG dispersion was measured in a sealed cell with Kapton windows. The sample-to-detector distance (SDD) was $110.0 \mathrm{~mm}$. Scattering data recorded on Kapton foils with the same experimental setup were used for background subtraction. The UKP-E and the NG paper were mounted on an open sample holder with $\mathrm{SDD}=110.6$ $\mathrm{mm}$.

According to paper testing standards (ISO 536, ISO 187), the handsheets were conditioned at $23{ }^{\circ} \mathrm{C}$ and $50 \% \mathrm{RH}$ for $24 \mathrm{~h}$ before the grammage, thickness, and mechanical properties were determined. The thickness was measured with a Lorentzen \& Wettre Micrometer 251 apparatus.

Strips of NG paper and reference paper were cut and tested in a Lorentzen \& Wettre tensile tester 066 to measure the tensile strength, tensile stiffness, and Young's modulus. The tensile strength and the tensile stiffness were also indexed against the grammage.

Thermogravimetric analysis (TGA) was performed to define the mass ratio of NG and UKP-E fibers in the paper. Samples of dried NG dispersion, dried UKP-E, and NG paper were heated from 30 to 900 ${ }^{\circ} \mathrm{C}$ using a Mettler Toledo apparatus at a heating rate of $20 \mathrm{~K} / \mathrm{min}$ in an ambient atmosphere. The revealed mass ratio of NG and UKP-E fibers was combined with the oven-dry grammage and the wet mix mass ratio to calculate the retention of the NG.

Cross-section samples of the paper were prepared using a Leica histological microtome. The cross-sections were imaged using a scanning electron microscope (SEM) (Zeiss, EVO50 LaB6Tip) and energy-dispersive X-ray spectroscopy (EDX) (Bruker, Quantax 200 SDD $30 \mathrm{~mm}^{2}$ ). On the obtained X-ray maps, oxygen was used as a marker for the UKP-E fibers, and carbon was used as a marker for the NG.

Individual fibers from an NG paper sample dispersed in distilled water were studied using a Sigma 500 Zeiss scanning electron microscope. The NG paper suspension was washed repeatedly in distilled water to remove the released NG. Individual fibers from this suspension were put onto an SEM sample holder, dried in oven for 5 min at $100{ }^{\circ} \mathrm{C}$, and cooled to room temperature before being mounted into the microscope.

The surface roughness was assessed with surface imaging using a Sensofar (PLux neox) profilometer with $20 \times$ magnification confocal lens. Both the NG paper as well as a reference paper were studied.

The surface free energy of the paper was derived from contactangle measurements with water and diiodomethane using a Krüss mobile surface analyzer.

Preparation of the ECD. The ECDs were prepared by screenprinting electrolyte ink and conducting ink. The screen with a 77-48 mesh size was masked with aluminum foil and distanced $10 \mathrm{~mm}$ from the printing substrate with rubber pads. Ink containing poly(3,4ethylenedioxythiophene) poly(styrenesulfonate) (PEDOT:PSS), purchased from Heraeus Clevios (Germany), was printed onto an indium-tin-oxide (ITO)-coated polyethylene terephthalate (PET) substrate and cured in oven $\left(110^{\circ} \mathrm{C}, 5 \mathrm{~min}\right)$. The electrolyte ink, kindly supplied by Rise Acreo (Sweden), was printed onto the smoother side of the NG paper using a screen with a mesh size of 5464. The two printed substrates were mounted with PEDOT:PSS toward electrolyte. 


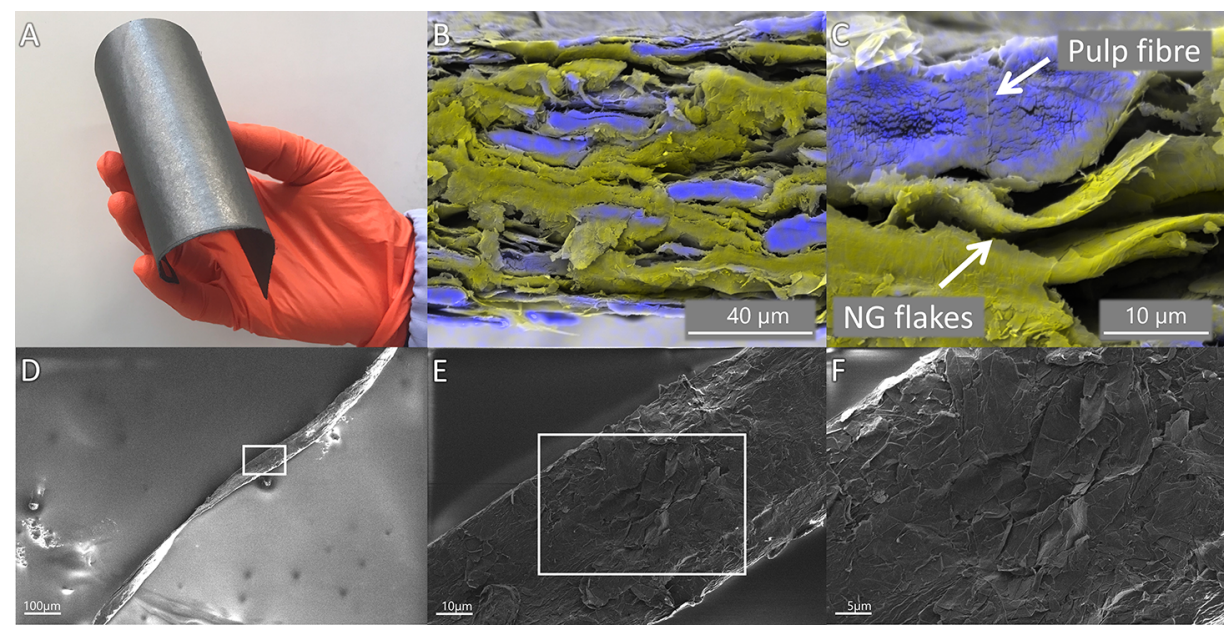

Figure 2. Characterization of the resulting NG paper (A), which appeared as a dark-gray, metallic-shining, nondusting, and flexible paper. SEM/ EDX images of the NG paper in the cross-section with (B) $520 \times$ and (C) $1755 \times$ magnification, where cellulosic fibers were identified with the presence of oxygen (blue) and NG with carbon only (yellow). SEM images of a redispersed fiber from the NG paper at (D) 100×, (E) 1000×, and (F) 2000 $\times$ magnification revealed a water-stable coating of NG flakes covering the entire fiber surface. The white rectangles represent the magnified area.

Electrical Measurements. Using a $3 \mathrm{D}$ four-point probe measurement technique, ${ }^{20}$ the in- and through-plane electrical conductivity of the paper sheet were measured. The measurements were carried out on a $30 \times 30 \mathrm{~mm}$ piece. A set of currents was applied in each of the two different planes, and the output voltage was measured. The calculated resistance was combined with the thickness measurements to calculate the conductivity of the paper.

Because a possible application of the NG paper could be flexible electronics, the electrical response to bending or folding was investigated. Using an ohmmeter with clip probes, the electrical resistance of the paper was measured before, during, and after bending and folding.

The durability toward different $\mathrm{pH}$ values and solvents common in inks and coating formulations was tested. Strips of the NG paper were bent down into five separate $50 \mathrm{~mL}$ beakers. Each beaker was then filled with $10 \mathrm{~mL}$ of $0.25 \mathrm{M}$ citric acid $\left(\mathrm{CA}_{\mathrm{ag}}\right)$, distilled water, $0.1 \mathrm{mM}$ potassium hydroxide $\left(\mathrm{KOH}_{\mathrm{aq}}\right)$, ethanol, and cyclopentanone, respectively. After $10 \mathrm{~min}$ of immersion, the paper strips were collected and dried on a hot plate heated to $70{ }^{\circ} \mathrm{C}$. Using an ohmmeter with pointed probes, the resistance of the paper was measured with a probe distance of $5 \mathrm{~cm}$ before and after the treatments with liquids.

The specific capacitance of the NG paper was measured by cyclic voltammetry, using a set of scan rates in a three-electrode setup with carbon felt as a counter electrode, silver/silver chloride $(\mathrm{Ag} / \mathrm{AgCl})$ as a reference, and $1 \mathrm{M}$ aqueous sodium chloride $\left(\mathrm{NaCl}_{\mathrm{aq}}\right)$ as an electrolyte. A potential window of $0-600 \mathrm{mV}$ versus $\mathrm{Ag} / \mathrm{AgCl}$ was used to minimize the interference from unwanted electrochemical redox reactions in the electrolyte.

The display performance was measured using an Ivium potentiostat. The switch time of the display was visually assessed in both directions, and the current-voltage characteristics were graphically recorded. The stability of the display was assessed by recording the current-voltage characteristics in an open circuit after the initial reduction. The device capacitance was characterized by chronopotentiometry (charge-discharge measurements) using a constant current of $0.1 \mathrm{~mA}$ and driving the cell voltage to $1 \mathrm{~V}$.

\section{RESULTS AND DISCUSSION}

WAXS Characterization. The measurements on the NG samples gave a distinct signal at $2 \theta=26.3^{\circ}\left(q=18.5 \mathrm{~nm}^{-1}\right)$ (Figure S1), characteristic of the (002) plane in graphite, ${ }^{21}$ indicating a highly crystalline material. The WAXS data recorded on the UKP-E sample showed a similar diffractogram as previous reports on unbleached kraft pulp, ${ }^{22}$ with characteristic diffraction peaks of cellulose I at $2 \theta=22.3^{\circ}(q=16.0$ $\left.\mathrm{nm}^{-1}\right)$ of the (200) plane and at $2 \theta=\sim 15^{\circ}\left(q=\sim 11 \mathrm{~nm}^{-1}\right)$ of the (110) plane (Figure S1).

The NG paper sample displays both the (002) signal from the NG and the (200) and (110) signals from the UKP-E sample (Figure S2). Hence the paper-making process has not significantly altered the crystal structure of the starting components.

Mechanical Properties and Retention Behavior. The resulting NG paper had a grammage of $78.8 \mathrm{~g} / \mathrm{m}^{2}$ and a thickness of $98.5 \mu \mathrm{m}$. It appeared as a dark-gray paper having a metallic-shining surface appearance, which did not dust and had the typical flexibility expected from an ordinary sheet of paper (Figure 2A).

Using a paper tensile tester, the mechanical properties of the NG paper were compared with a reference paper that was prepared using the same process but without containing any NG filler. As expected, the tensile index was lower when using a filler ( 16.75 vs $32.40 \mathrm{~N} \mathrm{~m} / \mathrm{g}$ for the reference paper) and is in line with the existing theory in paper mechanics. Interestingly, we observed a sharp increase in stiffness (Young's modulus reached 2.56 vs $1.02 \mathrm{GPa}$ for the reference paper), which is surprising because stiffness otherwise typically has an inverse correlation with the content of mineral fillers. ${ }^{23,24}$

From the cross-section elemental mapping images of the NG paper using SEM/EDX (Figure 2B,C), we found the NG flakes to appear as rather soft and flexible flaky structures that tend to bridge the cellulosic fibers. The NG flakes were clearly found to be located in between the fibers. Because the integrity in papers results from bonding between cellulosic fibers, the NG flakes should consequently considerably decrease the number of fiber-fiber bonding sites. This further makes the increase in tensile stiffness surprising, as the tensile stiffness should decrease with a lower number of fiber-fiber bonding sites. ${ }^{25}$ Still, it was initially assumed that parts of the fiber surfaces could potentially expose spots free from NG flakes, which then would provide available bonding sites between the cellulosic fibers. 
To investigate the presence of the NG-free fiber surface area available for direct fiber-fiber bonding, single fibers of the NG paper were imaged using SEM (Figure 2D-F). The redispersed and washed fibers from the NG paper appeared to the eye as tiny black needles. The SEM images reveal that the washed fibers are completely coated by the NG flakes, with a surface morphology totally different from that of the reference fibers (Figure S3). Because the coating of the fibers remained after the washing step, the NG flakes have a strong water-stable adhesion to the cellulosic fiber surfaces.

Several previous reports have presented stable formulations between CNFs and conducting nanocarbons; ${ }^{6,8}$ however, to our knowledge, no previous reports have described stable amalgamations between ordinary pulp fibers and carbon conductors. In the case of CNFs, several different explanations have been reported for the adhesion phenomena. One suggested mechanism is van der Waals forces operating between hydrophobic sites of the CNFs and the carbon material, ${ }^{4,5}$ whereas another suggestion is an interlocking effect caused by the induced polarization along the nanocarbons generated from the charged groups of the CNFs and their counterions. ${ }^{17}$ We view it as probable that the NG-fiber adhesion is of similar origins.

Regardless of what explains the NG-fiber adhesion, the extensive degree of NG coating around the cellulosic fibers shows that direct fiber-fiber bonding in the paper is at a very low level and implies that the integrity of the paper must result from mechanisms involving the NG coatings rather than direct fiber-fiber bonding. One plausible explanation could include restacking of the exfoliated graphite flakes ${ }^{26}$ to form strong interflake bonds, which hold the paper bulk together. This theory is supported by the considerable increase in the stiffness of the NG paper in at least two ways. First, graphite has a high inherent stiffness, ${ }^{27}$ which could be expressed in the paper properties. Second, given that the NG flakes form a strong adhesion to the fibers, the NG flakes could bridge the individual fibers and act as a cross-linker, indirectly increasing the fiber-fiber bonding degree. An increase in the fiber-fiber bonding degree is a common explanation for the increase in the tensile stiffness of papers when, for example, adding carboxy-methyl cellulose ${ }^{28}$ or a formula of cationic starch with mineral fillers. ${ }^{23,24}$

From a retention point of view, the attraction between the fibers and the NG filler is an attractive feature that enables high loadings without retention aid. Although the white water (i.e., the papermaking filtrate) was colored black from the excess $\mathrm{NG}$, the thermogravimetric characteristics of the resulting NG paper revealed a $50 \%$ mass loading of NG (Figure 3). The observed adhesion of the NG flakes to the fibers should be one explanation for such a high loading, although more factors must also be considered such as NG flake interactions and agglomeration $^{26}$ as well as a filtration mechanism provided by the cellulosic bulk fibers ${ }^{29}$ during the manufacturing process. However, although the loading is impressively high, the retention of the NG filler during the fabrication is only $50 \%$. To scale up the process, the filler recovery with white water recirculation, as done in commercial processes, should be more closely investigated to secure good production economics.

In papermaking, low drainage resistance is a key attribute to enable high production speed. The drainage resistance of the wet $\operatorname{mix}\left(49^{\circ} \mathrm{SR}\right)$ was higher compared to the reference pulp (19 $\left.{ }^{\circ} \mathrm{SR}\right)$, indicating that the NG flakes significantly contribute. Nonetheless, a drainage resistance of $49^{\circ} \mathrm{SR}$ for the NG/UKP-

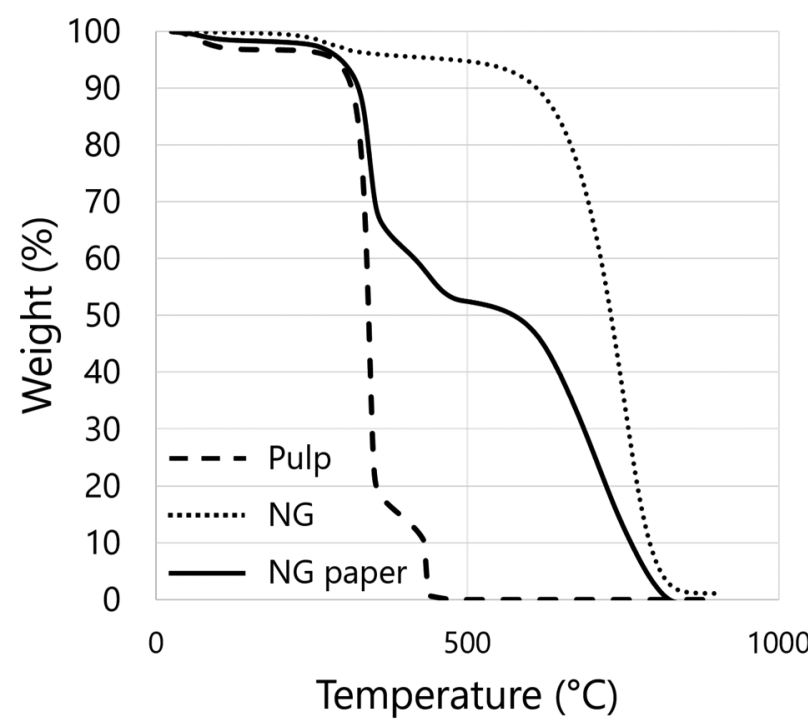

Figure 3. TGA graph for the NG paper and its components. The plateau for the $\mathrm{NG}$ paper starting at $500{ }^{\circ} \mathrm{C}$ corresponds to the more thermally stable NG filler.

E wet mix is still viewed as moderate $\left(100^{\circ} \mathrm{SR}\right.$ is equivalent to no drainage at all) and is not uncommon in papermaking.

Using an optical profilometer, the surface characteristics of the NG paper were compared with those of the reference paper. As expected, the NG flakes contribute to a smoother paper surface (Table S1). For the NG paper, the magnitude in roughness reduction depended on which side of the paper was measured, a difference not seen for the reference paper. The observed difference in the roughness of the NG paper should not be explained by the manufacturing procedure as such. Instead, these observations probably result from a nonuniform distribution of the NG filler in the vertical direction of the paper. In fact, this is a normal characteristic of filled papers, which is related to the unilateral dewatering direction in the drainage, ${ }^{30}$ rendering the filler material more concentrated toward one side of the paper. This indicates that fiber infiltration was a contributing mechanism to the NG flake retention, amid the good adhesion of the NG flakes to the UKP-E fiber surfaces.

Electrical Properties. Because graphite flakes are inherent 2D electronic systems, macroscopic conductors based on such conducting fillers could potentially exhibit anisotropic conductivity, although previous works on pulp/graphite composites have not encompassed this aspect. ${ }^{10,11}$ When measuring the electrical conductivity of the plain NG paper using a 3D four-point probe measurement setup, ${ }^{20}$ the out-ofplane conductivity is $12 \mathrm{~S} / \mathrm{cm}$, which is similar to values in previous reports describing pulp/graphite composites. On the contrary, the measured in-plane conductivity reaches a value of $107 \mathrm{~S} / \mathrm{cm}$, thus showing that a clear anisotropic conductivity is obtained. From the SEM/EDX images of the NG paper crosssection, we find a preferential orientation of the NG flakes along the in-plane direction (Figure 2C). Apparently, this inplane orientation of the NG flakes has a crucial impact on the conductivity anisotropy in the resulting NG paper.

A conductive paper should be interesting for printed electronics. However, this depends on whether or not the paper can maintain its good conducting properties after being exposed to the $\mathrm{pH}$ and solvent conditions common in inks and coating formulations. For example, printed electrolytes could 

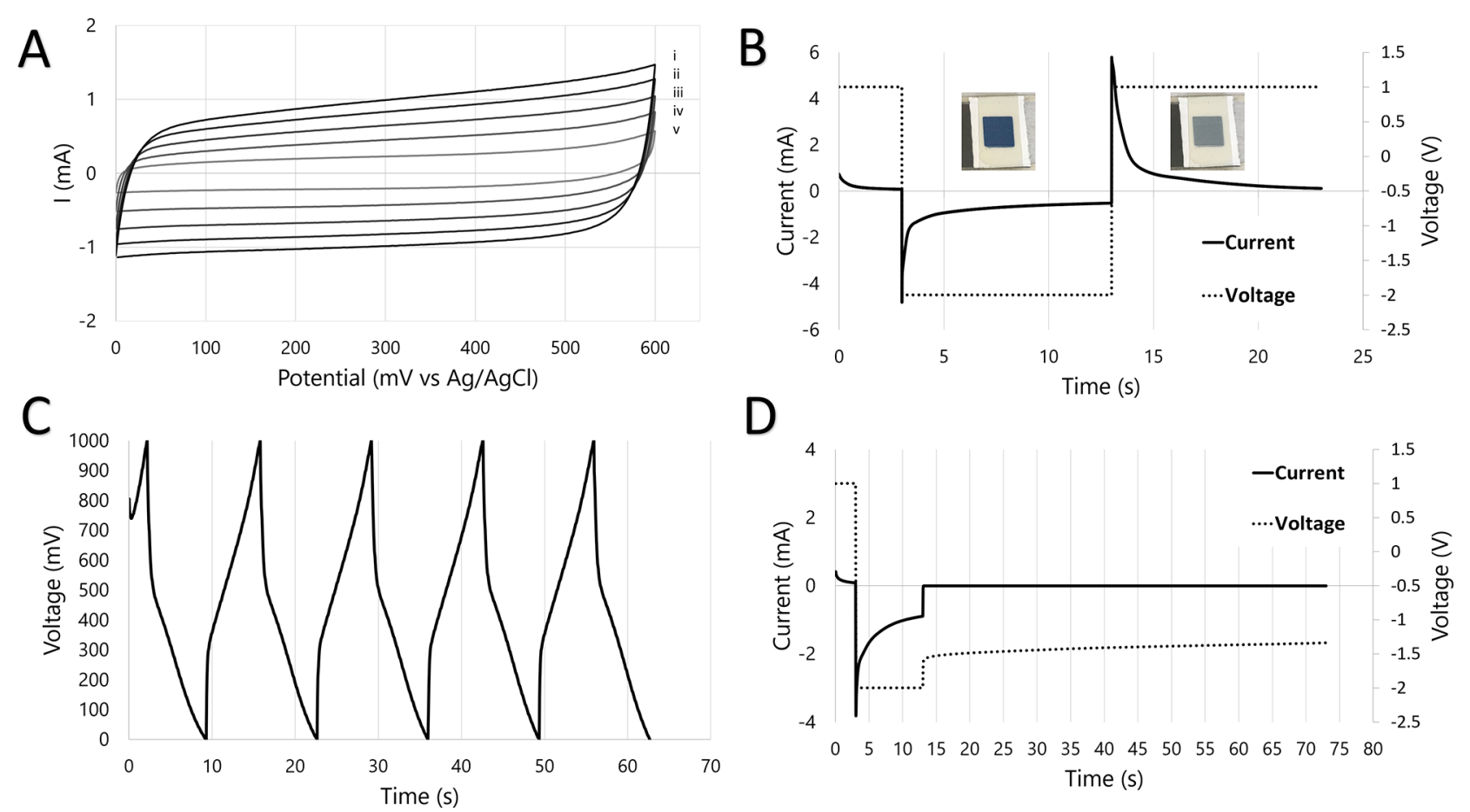

Figure 4. (A) Cyclic voltammetry of the $\mathrm{NG}$ paper in $1 \mathrm{M} \mathrm{NaCl}_{\mathrm{aq}}$ electrolyte using scan rates of 10, 20, 30, 40, and $50 \mathrm{mV} / \mathrm{s}(\mathrm{i}-\mathrm{v}) \mathrm{revealed}$ a unit area capacitance of $9.20 \mathrm{mF} / \mathrm{cm}^{2}$. (B) Current/voltage characteristics of the square-shaped ECD and the NG paper. The switch time assessment used an initial setting of the device for $3 \mathrm{~s}$, after which the PEDOT was reduced into its dark-blue color, followed by the reversed oxidation into its pale-blue state. (C) The charge/discharge curve of the square-shaped display revealed a device unit area capacitance of $1.04 \mathrm{mF} / \mathrm{cm}^{2}$. (D) The selfdischarge/bistability assessment was done in the open circuit for $60 \mathrm{~s}$ after reducing the PEDOT.

have a low $\mathrm{pH}$, coatings could be alkaline, and solvents such as plain water, alcohols like ethanol, or nonpolar solvents like cyclopentanone are common. Therefore, the electrical resistivity in strips of the NG paper was measured before and after being subjected to each of these conditions. Whereas no NG leaked into the solvents (Figure S4), the results show an increase in NG paper resistance for the aqueous solutions, with indications of an increased sensitivity toward a higher $\mathrm{pH}$ (Figure S5). This could be attributed to the swelling and shrinking of the pulp fibers upon wetting and drying, resulting in a rearrangement of the fibers. ${ }^{31}$ This could possibly also reorient and decouple the NG flakes, leading to the increased resistance. However, ethanol and cyclopentanone did not affect the electrical resistance of the paper. These solvents do not swell the fibers, so the paper integrity should be stable, which is manifested in the unchanged resistance of the NG paper.

Flexible electronics should also maintain their properties upon bending or even folding. The electrical resistance in a NG paper strip was therefore measured before and after folding it with an estimated bending radius of $1 \mathrm{~mm}$ (Figure S6). Interestingly, the resistance of the paper remained unchanged, demonstrating the suitability of this material for flexible electronics.

The specific double-layer capacitance of the NG paper, calculated from the responses in cyclic voltammetry, gave a resulting unit area capacitance of $9.20 \mathrm{mF} / \mathrm{cm}^{2}$ (Figure 4A). In relation to the grammage of the paper, this corresponds to a gravimetric capacitance of $1.17 \mathrm{~F} / \mathrm{g}$. For energy-storage applications, this is low compared with other carbon-based electrodes, whereas the electrical conductivity is unusually high. ${ }^{32,33}$ Applications for the NG paper should therefore be sought where this combination of properties is useful and, in particular, where mechanical stability and up-scalable production are of interest.

Printed Electronics Application. Printed ECDs are devices that could be considered as a good example fit to the properties of this paper. High conductivity is needed for a fast switch time, a reasonable capacitance is desired for display bistability, and a smooth surface is required for a fair printing performance. To explore the paper as a functionalized substrate according to the scheme in Figure 1B, the ECDs were prepared following a screen-printing manufacturing protocol. The aqueous polyelectrolyte ink wetted the paper surface well, indicating a hydrophilic surface. This was supported by surface energy measurement results from a mobile surface analyzer/goniometer, which revealed the NG paper to have a $76.8^{\circ}$ static contact angle to water and a surface free energy of $47.3 \mathrm{mN} / \mathrm{m}$ (polar component $3.79 \mathrm{mN} / \mathrm{m}$, dispersive component $43.55 \mathrm{mN} / \mathrm{m})$. The PEDOT-based electrochromic ink was printed onto a transparent ITO-coated PET foil, which then was manually laminated toward electrolyte-coated NG paper, with the electrochromic layer facing the electrolyte.

The electrochromic switching performance of these ECDs were evaluated using a potentiostat setup, operated in a constant voltage mode. The top PEDOT-based electrochromic electrode was reduced by applying a potential of $-2 \mathrm{~V}$, with respect to the NG paper counter electrode, for $10 \mathrm{~s}$. Following this, the PEDOT was then oxidized by applying $1 \mathrm{~V}$ for $10 \mathrm{~s}$ (Figure 4B). The time it took to switch from semitransparent (oxidized state) to dark blue (neutral state) was $\sim 1 \mathrm{~s}$, which corresponds to a drop in the current of $\sim 80 \%$. This is in good agreement with the current switching behavior of archetypical organic-based ECD devices. ${ }^{34-36}$ 
Using charge-discharge measurements with a constant current of $0.1 \mathrm{~mA}$, recorded from the square-shaped ECD, the net unit area capacitance of the ECD device was found to be $1.04 \mathrm{mF} / \mathrm{cm}^{2}$ (Figure $4 \mathrm{C}$ ). The shape is not exactly triangular due to the $i R$ drop, which is caused by the internal resistance of the device. Considering the NG paper unit area capacitance of $9.20 \mathrm{mF} / \mathrm{cm}^{2}$, the capacitance of the PEDOTbased top electrode can then be calculated to $1.17 \mathrm{mF} / \mathrm{cm}^{2}$. Thus the NG paper has a relatively much higher capacitance than the PEDOT-based electrode, meaning that the NG paper electrode does not capacitively limit the switch contrast of the PEDOT phase of the manufactured ECD.

Using the same equipment, the color and charge bistability of the ECD were evaluated. When the circuit was left open, the voltage instantly dropped to about $-1.5 \mathrm{~V}$ due to the internal resistance of the device, followed by a relatively much slower voltage loss (Figure 4D). According to the eye, the coloration of the displays seemed stable without any major fading in coloration and with no crosstalk between neighboring ECDs (Figure 5). We conclude that the capacitance of the NG paper is large enough to provide an ECD with good switching properties.

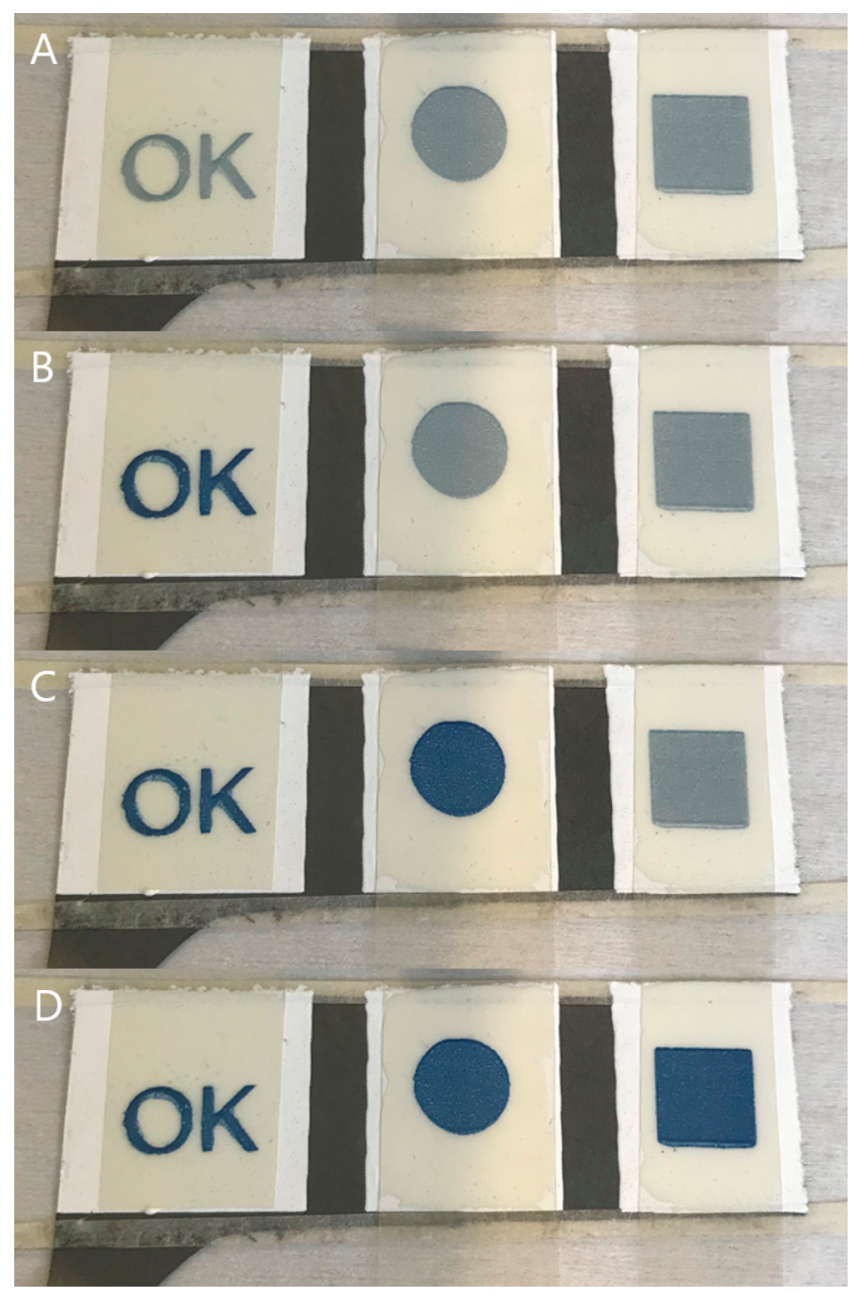

Figure 5. Electrochromic switching of the ECD. A set of printed electrochromic PEDOT:PSS figures, assembled on the same dark-gray NG paper, were switched (reduced) one after the other (A-D). The white areas are the printed electrolytes, and the transparent foil on top is the ITO-coated PET foil.

\section{CONCLUSIONS AND OUTLOOK}

Highly conducting papers can be easily prepared by using a standardized papermaking protocol with an acceptable drainage rate using electrotechnical unbleached pulp and an aqueous dispersion of NG flakes. The resulting paper also exhibits a reasonable specific capacitance, making it suitable as the backplane electronic paper for printed and flexible electronics, here demonstrated to perform well as the combined counter electrode, conductor, and substrate in a printed ECD. Owing to the excellent electrical and printability characteristics of the paper, the total number of layers to produce the resulting functional display component was reduced from seven to five.

The NG filler provides a reduction in the surface roughness and an increase in Young's modulus. The good retention of the NG filler is explained with infiltration by the fibers, but SEM images also reveal a water-stable adhesion of NG to cellulosic fibers. This coating of the fibers with NG challenges the established paper physics, as the paper integrity cannot be explained by ordinary fiber-fiber bonding. Although the interactions need to be better understood and optimized, our findings demonstrate a successful pathway to realize biobased electronic materials using standard manufacturing protocols.

We believe that NG paper will find its way into numerous large-volume applications in flexible and printed electronics due to its up-scalability, favorable mechanical properties, and stability and the combination of high conductivity along with charge-storage ability. For instance, it can potentially be used as a large-area and porous electrode in various electrocatalytic and electrochemical setups targeting the production of fuels, and in fuel cells, it can act as the dually conducting and capacitive electrode in transistors, and it can also serve as the electrodes in batteries and supercapacitors, especially because it maintains a high conductivity at different potentials.

\section{ASSOCIATED CONTENT}

\section{SI Supporting Information}

The Supporting Information is available free of charge at https://pubs.acs.org/doi/10.1021/acsami.0c13086.

WAXS results, SEM images of reference and NG paper pulp fibers, solution resistance results, folding test, topography imaging, and surface roughness (PDF)

Switching of the printed ECD (MP4)

\section{AUTHOR INFORMATION}

\section{Corresponding Authors}

Isak Engquist - Laboratory of Organic Electronics, Department of Science and Technology, Linkoping University, 60174 Norrkoping, Sweden; 이이. orcidoo00-0001-5365-6140; Email: isak.engquist@liu.se

Magnus Berggren - Laboratory of Organic Electronics, Department of Science and Technology, Linkoping University, 60174 Norrkoping, Sweden; Email: magnus.berggren@liu.se

\section{Authors}

Patrik Isacsson - Laboratory of Organic Electronics, Department of Science and Technology, Linkoping University, 60174 Norrkoping, Sweden; 다이. org/0000-0002-74883788

Xin Wang - RISE Digital Systems, Department of Smart Hardware, 60174 Norrkoping, Sweden 
Andreas Fall - RISE Bioeconomy and Health, Department of Pulp, Paper and Packaging, 11486 Stockholm, Sweden

Desalegn Mengistie - Laboratory of Organic Electronics, Department of Science and Technology, Linkoping University, 60174 Norrkoping, Sweden

Emilie Calvie - Ahlstrom-Munksjö Research Center, 38140 Apprieu, France

Hjalmar Granberg - RISE Bioeconomy and Health, Department of Pulp, Paper and Packaging, 11486 Stockholm, Sweden

Göran Gustafsson - RISE Digital Systems, Department of Smart Hardware, 60174 Norrkoping, Sweden

Complete contact information is available at: https://pubs.acs.org/10.1021/acsami.0c13086

\section{Author Contributions}

The manuscript was written through contributions of all authors.

\section{Funding}

This work was funded by the Digital Cellulose Centre, a competence center set up by the Swedish Innovation Agency VINNOVA and a consortium of Swedish forest industries. Additional funding has been provided by the Wallenberg Wood Science Center (Knut and Alice Wallenberg Foundation), the VINNOVA "EPIC" project (2017-05413 Elektrooch fotokatalys inducerat i papper), and the Karl-Erik Önnesjö Foundation.

\section{Notes}

The authors declare no competing financial interest.

\section{ACKNOWLEDGMENTS}

The pulp was kindly supplied by Ahlstrom-Munksjö Billingsfors mill, Sweden, which also let us use their papermaking equipment and provided the measurement of the drainage resistance. SEM/EDX analysis was done by the AhlstromMunksjö analysis team in Apprieu, France. The NG used was kindly supplied by 2D fab in Sundsvall, Sweden. The WAXS characterizations were carried out at RISE Bioeconomy by Anita Teleman. We also acknowledge the support from Treesearch, a collaboration platform for Swedish forest industrial research.

\section{REFERENCES}

(1) OE-A Roadmap for Organic and Printed Electronics, 8th ed.; Organic and Printed Electronics Association: Frankfurt, Germany, 2020.

(2) Lin, Y.; Gritsenko, D.; Liu, Q.; Lu, X.; Xu, J. Recent Advancements in Functionalized Paper-Based Electronics. ACS Appl. Mater. Interfaces 2016, 8, 20501-20515.

(3) Pawlicka, A. Development of Electrochromic Devices. Recent Pat. Nanotechnol. 2009, 3, 177-181.

(4) Xiong, R.; Kim, H. S.; Zhang, L.; Korolovych, V. F.; Zhang, S.; Yingling, Y. G.; Tsukruk, V. V. Wrapping Nanocellulose Nets around Graphene Oxide Sheets. Angew. Chem., Int. Ed. 2018, 57, 8508-8513.

(5) Ferreira, E. S.; da Silva, D. S.; Burgo, T. A. L.; Batista, B. C.; Galembeck, F. Graphite Exfoliation in Cellulose Solutions. Nanoscale 2017, 9, 10219-10226.

(6) Hamedi, M. M.; Hajian, A.; Fall, A. B.; Håkansson, K.; Salajkova, M.; Lundell, F.; Wågberg, L.; Berglund, L. Highly Conducting, Strong Nanocomposites Based on Nanocellulose-Assisted Aqueous Dispersions of Single-Wall Carbon Nanotubes. ACS Nano 2014, 8, $2467-2476$.
(7) Osong, S. H.; Dahlström, C.; Forsberg, S.; Andres, B.; Engstrand, P.; Norgren, S.; Engström, A.-C. Nanofibrillated Cellulose/Nanographite Composite Films. Cellulose 2016, 23, 2487-2500.

(8) Yang, W.; Zhang, Y.; Liu, T.; Huang, R.; Chai, S.; Chen, F.; Fu, Q. Completely Green Approach for the Preparation of Strong and Highly Conductive Graphene Composite Film by Using Nanocellulose as Dispersing Agent and Mechanical Compression. ACS Sustainable Chem. Eng. 2017, 5, 9102-9113.

(9) Peng, Y.; Gardner, D. J.; Han, Y. Drying Cellulose Nanofibrils: In Search of a Suitable Method. Cellulose 2012, 19, 91-102.

(10) Andres, B.; Dahlström, C.; Blomquist, N.; Norgren, M.; Olin, H. Cellulose Binders for Electric Double-Layer Capacitor Electrodes: The Influence of Cellulose Quality on Electrical Properties. Mater. Des. 2018, 141, 342-349.

(11) Jabbour, L.; Chaussy, D.; Eyraud, B.; Beneventi, D. Highly Conductive Graphite/Carbon Fiber/Cellulose Composite Papers. Compos. Sci. Technol. 2012, 72, 616-623.

(12) Hubbe, M. A.; Heitmann, J. A. Review of Factors Affecting the Release of Water from Cellulosic Fibers during Paper Manufacture. BioResources 2007, 2, 500-533.

(13) Montibon, E.; Lestelius, M.; Järnström, L.; Love, K. T. Paper Physics: Electroconductive Paper - A Study of Polymer Deposition and Conductivity Influenced by Sheet Forming and Fibre beating. Nord. Pulp Pap. Res. J. 2010, 25, 473-480.

(14) Goto, H. Electrically Conducting Paper from a Polyaniline/ Pulp Composite and Paper Folding Art Work for a 3D Object. Text. Res. J. 2011, 81, 122-127.

(15) Yarar Kaplan, B.; Isıkel Sanl1, L.; Alkan Gursel, S. Flexible Carbon-Cellulose Fiber-Based Composite Gas Diffusion Layer for Polymer Electrolyte Membrane Fuel Cells. J. Mater. Sci. 2017, 52, 4968-4976.

(16) Laine, J.; Stenius, P.; Carlsson, G.; Ström, G. The Effect of ECF and TCF Bleaching on the Surface Chemical Composition of Kraft Pulp as Determined by ESCA. Nord. Pulp Pap. Res. J. 1996, 11, 201210.

(17) Hajian, A.; Lindström, S. B.; Pettersson, T.; Hamedi, M. M.; Wagberg, L. Understanding the Dispersive Action of Nanocellulose for Carbon Nanomaterials. Nano Lett. 2017, 17, 1439-1447.

(18) Blomquist, N.; Engström, A.-C.; Hummelgård, M.; Andres, B.; Forsberg, S.; Olin, H. Large-Scale Production of Nanographite by Tube-Shear Exfoliation in Water. PLoS One 2016, 11, e0154686.

(19) Stark, H.; Eichinger, R.; Stecher, W. Effect of Filler Characteristics on Retention and Sheet Strength. Wochenblatt für Papierfabrication 1984, 112, 409-415.

(20) Wang, X.; Grimoldi, A.; Håkansson, K.; Fall, A.; Granberg, H.; Mengistie, D.; Edberg, J.; Engquist, I.; Nilsson, D.; Berggren, M.; Gustafsson, G. Anisotropic Conductivity of Cellulose-PEDOT:PSS Composite Materials Studied with a Generic 3D Four-Point Probe Tool. Org. Electron. 2019, 66, 258-264.

(21) Edberg, J.; Brooke, R.; Hosseinaei, O.; Fall, A.; Wijeratne, K.; Sandberg, M. Laser-Induced Graphitization of a Forest-Based Ink for Use in Flexible and Printed Electronics. npj Flex. Electron. 2020, 4, No. 17.

(22) Agarwal, U.; Reiner, R.; Ralph, S. Estimation of Cellulose Crystallinity of Lignocelluloses Using Near-IR FT-Raman Spectroscopy and Comparison of the Raman and Segal-WAXS Methods. J. Agric. Food Chem. 2013, 61, 103-113.

(23) Lindström, T.; Floren, T. The Effects of Cationic Starch Wet End Additions on the Properties of Clay Filled Papers. Nord. Pulp Paper. Res. J. 1984, 87, 97-104.

(24) Tanaka, A.; Hiltunen, E.; Kettunen, H.; Niskanen, K. InterFiber Bonding Effects of Beating, Starch or Filler. Nord. Pulp Pap. Res. J. 2001, 16, 306-312.

(25) Alava, M.; Niskanen, K. The Physics of Paper. Rep. Prog. Phys. 2006, 69, 669-723.

(26) Stankovich, S.; Piner, R. D.; Chen, X.; Wu, N.; Nguyen, S. T.; Ruoff, R. S. Stable Aqueous Dispersions of Graphitic NanoPlatelets via the Reduction of Exfoliated Graphite Oxide in the Presence of Poly(sodium-4-styrenesulfonate). J. Mater. Chem. 2006, 16, 155-158. 
(27) Golkarian, A. R.; Jabbarzadeh, M. The Density Effect of van der Waals Forces on the Elastic Modules in Graphite Layers. Comput. Mater. Sci. 2013, 74, 138-142.

(28) Blomstedt, M.; Kontturi, E.; Vuorinen, T. Optimising CMC Sorption in Order to Improve Tensile Stiffness of Hardwood Pulp Sheets. Nord. Pulp Pap. Res. J. 2007, 22, 336-342.

(29) Sampson, W. W.; Turner, P. J. The Effect of Suspension Crowding on the In-Plane Distribution of Filler in Handsheets. Appita J. 1996, 49, 274-276.

(30) Solberg, D.; Wagberg, L. On the Mechanism of GCC Filler Retention During Dewatering - New Techniques and Initial Findings. J. Pulp Paper Sci. 2002, 26, 183-188.

(31) Grignon, J.; Scallan, A. Effect of $\mathrm{pH}$ and Neutral Salts Upon the Swelling of Cellulose Gels. J. Appl. Polym. Sci. 1980, 25, 2829-2843.

(32) Portet, C.; Yushin, G.; Gogotsi, Y. Electrochemical Performance of Carbon Onions, Nanodiamonds, Carbon Black and Multiwalled Nanotubes in Electrical Double Layer Capacitors. Carbon 2007, 45, 2511-2518.

(33) Dubey, R.; Guruviah, V. Review of Carbon-Based Electrode Materials for Supercapacitor Energy Storage. Ionics 2019, 25, 14191445.

(34) Cai, G.; Cheng, X.; Layani, M.; Tan, A. W. M.; Li, S.; Eh, A. L.S.; Gao, D.; Magdassi, S.; Lee, P. S. Direct Inkjet-Patterning of Energy Efficient Flexible Electrochromics. Nano Energy 2018, 49, 147-154.

(35) Kawahara, J.; Ersman, P. A.; Engquist, I.; Berggren, M. Improving the Color Switch Contrast in PEDOT:PSS-Based Electrochromic Displays. Org. Electron. 2012, 13, 469-474.

(36) Santos, G. H.; Gavim, A. A. X.; Silva, R. F.; Rodrigues, P. C.; Kamikawachi, R. C.; de Deus, J. F.; Macedo, A. G. Roll-to-Roll Processed PEDOT:PSS Thin Films: Application in Flexible Electrochromic Devices. J. Mater. Sci.: Mater. Electron. 2016, 27, 1107211079 . 